\section{CLINICAL LECTURE ON}

\section{CASE OF INFLAMMATORY EFFUSION INTO THE PLEURA AND PERITONEUM: PARACENTESIS THORACIS : RECOVERY: WITH CLINICAL REMARKS.}

By C. HANDFIELD JONES, M.B.Cantab., F.R.S., Physician to St. Mary's Hospital.

C. S., aged 19, engineer, was admitted July 20th, 1869. His health had been generally good; he had never been ill before. His mother, he thought, died of phthisis. He had been ill about fourteen days. His abdomen swelled and felt tight, as it was on admission. He had had no diarrhcea, but the bowels had acted with medicine. The urine was scanty, thick, and dark; pulse 96 , weak. The heart's sounds were normal. He had fairly good breathing, though a little harsh in both upper fronts; good breathing in all the left lung, but very distinct small crepitations in the right lower back. He had some cough, nearly dry the last week. The abdomen was equally distended, resonant at the right side and middle, but dull in the left side in the iliac region and flank, and up to the posterior part of the left hypochondrium. Altering his position did not affect the dulness much, but pressure on the dull part before percussion produced a degree of tympanitic resonance. The abdomen was quite painless. The mental faculties were quite clear. Respirations 22; temperature $100.2 \mathrm{deg}$. He was ordered two grains of blue pill and a grain of powdered digitalis in a pill three times a day; a warm bath; half a drachm of bitrate of potash in an electuary three times a day; and broth-diet.-July 24th. The pill was repeated, and he was ordered twenty grains of nitrate of potash in infusion of scoparium three times a day.-July 28 th. The quantity of fluid in the abdomen was evidently increased, as estimated by the extent of the dulness, which was now notable on the right as well as on left on light percussion. The circumference at the umbilicus was 32 inches. The urine was 22 ounces in quantity in twenty-four hours; it deposited lithates. He felt well, except for the discomfort of his abdomen. He was ordered to have ointment of biniodide of mercury rubbed into the abdomen, and to take four grains of iodide of potassium in an ounce of water three times a day.-July 3 Ist. The skin of the abdomen was reddened, partly erythematous, partly papular. The urine was 28 ounces, of full colour. The superficial abdominal veins were full.-Aug. 4th. The ascites seemed rather less, but the left pleura was full of fluid. The heart was displaced to the right of the sternum, pulsating in the fourth and fifth spaces, just inside the vertical line of the right nipple. The dulness extended up to the left clavicle. There were dulness and bronchial breathing and voice in the left back. The right back was resonant; but at the base there was a good deal of small crepitation, aud some rhonchus higher up. There was no notable dyspncea. He lay down easily. Respirations 30; pulse 132, distinct temperature $97.6 \mathrm{deg}$. Paracentesis was about to be performed, but was deferred because distant lung-sounds were audible in the left side, and there was no urgency. He was ordered half a grain of opium four times a day, turpentine stupes to the side, and to continue the mixture. - Aug. 5th. The urine amounted to 42 ounces. He had two ounces of brandy. He felt quite cumfortable ; ate a chop, egg, and pudding, well.-Aug. 6th. The pulse was of much more volume and force; respirations 27 ; temperature $98.6 \mathrm{deg}$. The urine was clear, normallooking, acid, not albuminous, 33 ounces in quantity. All the left back was dull, but at the base breathing was heard loud; the inspiration somewhat vesicular, the expiration tubular, the voice bronchophonic. At the upper part of the back, breathing was loud, chiefly tubular. Below the left clavicle, the breathing was more normal. In the left side, the breathing was very weak and distant. The circumference of the abdomen was $30 \frac{1}{2}$ inches.-Aug. 8th. He was ordered to take the pill every three hours. - Aug. 9th. He had a very good night, and felt comfortable. Pulse 128, weak. The pleural effusion was rather increased. Paracentesis was performed, and 86 ounces of fluid removed. This fluid coagulated in twenty-four hours into a red-streaked fibrinous network floating in serum. Under the microscope, the fibrine showed homogeneous granular strands of good consistence, entangling multitudes of red corpuscles and numerous pale granule-cells, circular, of various sizes, some as large as an hepatic cell. Two test-glasses, into which some liquor ammoniæ had been put before they were filled, contained very little coagulum, and almost all the blood-corpuscles were destroyed; some filmy traces of corpuscles remained. The heart moved over to the left of the sternum while the fluid was flowing; I saw it distinctly to-day nearly in its normal site. In the left front, quasi-crepitation was heard as far down as the nipple, and weak breathing below the clavicle. In the side, friction-sound much resembling crepitation was heard about the middle, and distant breathsound. In the back, a friction-sound of very much the same character was heard, at the base and in almost all its extent; bronchaphony existed at the middle. Fremitus was distinct in the right back, null in the left. Pulse 105, weakish; respirations 24, quiet and regular. The mixture was omitted; and he was ordered to take sixteen minims of tincture of digitalis, and fifteen minims of solution of perchloride of iron, in an ounce of infusion of quassia, three times a day. On Aug. I Ith, the opium pill was omitted.-Aug. 14th. Pulse I IO. The right back was resonant; breathing was perfect in this region. In the left back there was dulness in the lower half; the breathing was weak, and there was a little grazing friction-sound. In the upper half of the left back there was good resonance and breathing. In the upper half of the left side, resonance was good; and very good breathing was heard down to the level of the sixth costal cartilage. There were good resonance and breathing in all the left front, with some indeterminate quasi-crepitant sounds. The ascites had nearly disappeared. The urine appeared very natural ; on the 13 th, it was 38 ounces. He continued to improve steadily. On the 25 th, there was full deep breathing at the posterior left base, and soon afterwards he left the hospital.

REMARKS. - The cause of the serous effusions in this case is very obscure. There can be no doubt that they came on in a gradual, quiet manner, without pain or any of the stormy symptoms of acute inflammation. It is also certain, I think, that they were not of renal origin. Though my notes do not actually state that the urine on admission was not albuminous, yet, as other particulars are mentioned, I am sure this most important one would not have been omitted from the investigation, and, had albumen been found, the fact would have been recorded. The urine was simply that of pyrexia-say of acute rheumatism. The temperature was moderate on admission, and was normal when the pleural effusion was near its acme. Internal medicines were of little or no avail ; but paracentesis thoracis had the best effects; the fluid speedily disappeared from both serous cavities, and complete recovery took place. This favourable influence of the removal of dropsical accumulation has often been noted, and was well illustrated in a case which I attended several times with a medical friend. The patient had copious left-side pleural effusion, causing great distress and dyspnoea. We operated three several times, drawing off some sixty or eighty ounces of fluid each time. Before the operation, the urine was always scanty, once or twice very much so; and the appetite almost null. Immediately after the operation, the urine became copious, and the appetite good; and this improvement continued some time, until the fluid accumulated again. I do not feel that I can satisfactorily explain this effect of paracentesis, so I shall content myself with commending the clinical fact to your notice : warning you, however, that it is very far from being an invariable result.

To one point in connexion with the paracentesis I would shortly advert; I mean the postponement of the operation five days on account of distant lung-sounds being heard in the region where we proposed to perforate the chest-wall. I certainly prefer to find no breath-sound in the spot where I am about to plunge my trochar; but I am satisfied that weak and distant breathing should not deter us, as it did me on that occasion. Lung-sounds can penetrate through a notable thickness of fluid. There is good reason not to delay the performance of paracentesis when it has become evident that other remedies are not likely to avail ; for every day that passes increases the liability of the lung to become bound down to the mediastinum by false membranes; and there is also the risk, if the accumulation of fluid be large, of fatal syncope occurring suddenly.

Let me now say a few words respecting the fluid which we drew off from the pleura. It was evidently not mere serum, but was rich in fibrine and corpuscles. The presence of fibrine assimilates it closely to liquor sanguinis, the fluid part of the blood in which the corpuscles float. The coagulation was evidently not dependent on the presence of corpuscles in a fibrinous fluid; for it did not take place as long as the fluid remained in the serous cavity, but occurred soon after its withdrawal from the vicinity of the living tissues. The same change occurs when a piece of inanimate matter is intruded into the living and circulating blood, as when a needle is passed through the coat of a vein, and is bathed in the blood-current for some time: on laying open the vein, the point of the needle is found coated with a deposit of fibrine. Next as to the corpuscles. It is clear that the red corpuscles must have proceeded from the blood: the only question is, whether they were accidentally mingled with the fluid from the slight bleeding necessitated by the operation, or whether they actually escaped from the pleural vessels 
ust as the fluid effusion itself did, I entertain no doubt myself that the latter was the case : the red cells were too numerous and too widely diffused to have gained admission accidentally. The non-coloured corpuscles were essentially similar to those met with in pneumonia and other inflammatory processes. Respecting their genesis, different views are held; some looking upon them, with Cohnheim, as white bloodcells which have traversed the walls of the vessels; others agreeing with Virchow and Stricker, that they are the progeny of altered tissue-cells. Both doctrines may be true; but, if I were to adopt one exclusively, it would be the first. For (I) it seems unquestionable that corpuscles do make their way readily enough, under certain circumstances, through the walls of capillaries ; so that the old belief in diapedesis, after having been scouted as absurd, is now re-established. (2) There is a striking resemblance between the intra- and extra-vascular cell-formations. The exudation which fills up the air-cells of a hepatised lung may consist to a very large extent of corpuscles almost identical with those which are found in coagula formed in the heart or vessels. I am not, of course, referring to the red corpuscles, but to the white and their developments. Some of these, found in intravascular clots, are altogether similar to the cells formed in inflammatory foci. When the white corpuscles have made their way into the interstices of the tissues, they very probably multiply and give rise to pus or other inflammatory cell-growth. Possibly the tissue-corpuscles may do the same; but this seems to me less certain.

It seems to me very difficult to believe that a large-probably the largest-part of the inflammatory products found on serous or mucous surfaces does not directly proceed from the blood, and is not, correctly speaking, an exudation. Fibrine or fibrinogen certainly exists in the blood, and is also certainly present in the uncoagulated state in anasarcous fluid, and in the ccpious effusions that are generated by pleurisy. The surface of inflamed serous membranes is often covered with more or less thick layers or masses of a substance which bears, in its general appearance, a very elose resemblance to pure fibrinous coagulum, and and which, as Rokitansky's and Paget's observations tend to show, varies in structural composition very much as the latter does. Recently, in a case of rheumatic pericarditis, I found the shaggy projections from the inflamed serous membrane to consist mainly of an homogeneo-granular blastema almost devoid of corpuscles. The latter were abundant amid the fibres of the inflamed membrane, and were pretty numerous on its surface; but it seemed to me quite impossible that the solid, bulky, overlying stratum was formed from altered cor. puscles. I could only regard it as an off-flow of that blood-element which is known to be in excess in states of inflammation, and which has only to traverse a most thin film of homogeneous membrane to reach the situation where it is found. In the case referred to, there was no pus in the pericardium, and no large amount of serous fluid. The fibrine of a cardiac clot appeared to be of very good quality; the fibrils were well formed and numerous; the granular matter rather scanty; and the corpuscles small and not numerous. I do not assert that the intra- and extra-vascular fibrine were identical ; but, having regard to all the circumstances, I cannot doubt that the latter proceeded from the former, or rather from the blood-constituent which produced the former. In catarrh of mucous membranes, the corpuscles by no means constitute all the inflammatory product ; there is also a more or less abundant intercellular substance, which is the characteristic product of the morbid process. This tenacious mucous substance is alkaline, like liquor sanguinis, and seems to be essentially this fluid-modified, however, in its transit through a cell-bearing surface. For, where this cell-layer is wanting, as in the pulmonary air-cells, and on denuded eczematous patches, the exudation (which in the latter case, at any rate, is alkaline) loses the mucous quality. These (old) views seem to me concordant with clinical experience of the value of remedies which lessen local hyperæmia or diminish the entire blood-mass, as in recent observations on abstinence from fluids in promoting reabsorption of pleuritic effusion.

A few words now as to the process which causes this exudation. Some miasm or morbific agent in the blood comes to operate on the part which happens to be at the time the least resisting, and deranges the vital actions of its component tissues. The small arteries relax, and increased local blood-flow ensues; the capillaries lose their normal retentive power, and allow at first fluid and then corpuscles to escape ; the tissue-elements exert an abnormal attraction on the blood-cells which accumulate in and obstruct the capillaries. This latter is the most important factor of inflammation. In proportion to its development, I conceive, is the severity of the disorder. Further, it seems probable that this derangement of the nutrition-power of the tissues is powerfully influential on the exudation itself. If it be but moderate, the cell-formations are capable of a low form of organisation; if it be excessive, they become effete and dead as regards the organism, and are cast off as pus. In this determination of the fate of the exudation, the quality of the blood is also materially influential. If its fibrine have a decided tendency to assume the corpuscular form, the conversion of the exudation into pus is greatly promoted.

In the case which I have narrated to you, the morbid process was doubtless inflammatory, but was not of great virulence. The exudation, though it certainly contained corpuscles undistinguishable from pus, was very far from being purulent. If we had had to do with an empyema, the recovery would have been vastly slower and more imperfect. You see, then, that inflammations are not all of the same quality. In the lowest degrees of the morbid process, as Mr. Paget states, we have serum alone effused; in the vast majority, we have liquor sanguinis more or less altered, and containing a varying amount of blood-corpuscles, chiefly the white. If the vital endowments of blood and tissues are not too much depressed by local or general conditions, the effused matters, as the morbid action ceases, return to the blood again -are reabsorbed. But, if the devitalising injury have been greater, or the resisting power more feeble, the exudation becomes more degraded, and is cast off as effete pus; and, if the morbid action be still more intense, the exudation and the tissues perish together in gangrene. Most commonly these several grades, which we have regarded as typically separate, are variously blended together.

\section{ABSTRACTS OF LECTURES ON THE}

GEOGRAPHICAL DISTRIBUTION OF DISEASES IN ENGLAND AND WALES.

Delivered at St. Thomas's Hospital, London.

By ALFRED HAVILAND, EsQ.

V.*

The Geographical Distribution of Phthisis in the 623 RegistrationDistricts.

As it would be impossible to enter minutely into the groups of districts within the hour, I shall now merely draw your attention to certain social, physical, climatic, and geographical facts, which are coincident with either the high or low mortality from phthisis in the 623 registration-districts of England and Wales.

The Distribution of Phthisis, compared with that of other Diseases. I have already alluded so frequently to the differences in distribution of heart-disease and that of phthisis, that I will only now revert to cancer, and shortly compare some of the more important features displayed in the two maps.

The great cancer-field of the Thames will be seen to be almost coextensive with its catchment basin, forming an irregular quadrilateral area of high mortality, and therefore coloured blue in the map of cancer. We see how the darkest districts are grouped around the course of the Thames. If we now examine the map of phthisis, we shall find this very four-sided blue area coloured red, indicating a very low mortality from consumption; in fact, it is the converse of what ob. tained in cancer. In this area will be found a remarkable group around London, all of the lowest mortality; London itself having a mortality of the fourth degree, giving us the idea that the immediate districts around London had poured their phthisical patients into the twenty-six London hospitals, thereby reducing their own death-rate, whilst they raised that of the metropolis. But, if the metropolitan figures were merged into those of its neighbours, the group would even then have a mortality of the lowest degree. This was not the case with cancer, as London had a lower death-rate from this cause than some of the immediate districts-Richmond, for instance.

The Yorkshire cancer-field follows, as we have seen, the courses of the oft-flooded rivers of this county. In the phthisis-map, we find the blue area coloured red, indicating the lowest mortality. In the West of England, if you trace the courses of the Severn and the Wye, you will find that their riparial districts are so coloured as to show a high death-rate from cancer. Compare this oblong area with the courses of these rivers in the phthisis-map, and you will find that the districts show very low death-rate. Again, compare the cancer-field of mid Devon with the same area in phthisis, and you will see as remarkable a contrast as any of those alluded to. I have dwelt upon this point because it is a remarkable and unexpected fact, and worthy of further study. When I was exhibiting my maps before the Royal Society, Mr. Erasmus Wilson was much struck with what I have now pointed out; and he

* Concluded from page 58. - The map illustrating this paper was published with the number of January 7 th. 